\title{
Original
}

\section{Criterios ecográficos de malignidad en adenopatías cervicales subclínicas de pacientes con carcinoma epidermoide oral}

\author{
Teresa Creo Martínez ${ }^{a, *}$, Yolanda Marín Lapeira ${ }^{b}$ y Ángel Rollón Mayordomo \\ ${ }^{a}$ Médico adjunto, Servicio de Cirugía Oral y Maxilofacial, Hospital Universitario Puerta del Mar, Cádiz, España. \\ ${ }^{b}$ Médico adjunto, Servicio de Radiología, Hospital Universitario Virgen Macarena, Sevilla, España. \\ 'Jefe de Sección, Servicio de Cirugía Oral y Maxilofacial, Hospital Universitario Virgen Macarena, Sevilla, España.
}

\section{INFORMACIÓN DEL ARTÍCULO}

\section{Historia del artículo:}

Recibido el 11 de mayo de 2010

Aceptado el 15 de noviembre de 2010

\section{Palabras clave:}

Metástasis cervicales;

Detección de metástasis con

ultrasonidos;

Carcinoma de cavidad oral;

Carcinoma epidermoide;

Estadificación cervical;

Detección precoz de adenopatías

ocultas;

Disección cervical

\section{R E S U M E N}

El carcinoma epidermoide de cavidad oral produce metástasis cervicales subclínicas en el 30-40\% de los casos, lo que justifica la disección cervical electiva profiláctica dentro del tratamiento. La disponibilidad de pruebas diagnósticas que nos permitiesen detectar dichas metástasis evitaría el sobretratamiento del 60-70\% de los pacientes, así como el coste y la morbilidad asociados. La ecografía cervical detecta las metástasis subclínicas con una sensibilidad y especificidad variables.

Objetivos: Usar la ecografía para la valoración de adenopatías cervicales metastásicas antes de plantear el tratamiento quirúrgico.

Material y método: Hemos realizado un estudio de seguimiento prospectivo de pacientes diagnosticados de carcinoma epidermoide de cavidad oral en estadio precoz (I-II) a los que se les ha efectuado un estudio ecográfico cervical (con longitud de onda larga) antes del tratamiento quirúrgico. Hemos revisado y aplicado diferentes criterios de malignidad obtenidos de los estudios publicados. Hemos comparado los datos de la ecografía con los resultados del estudio anatomopatológico de la disección cervical que hemos usado como patrón oro de metástasis.

Resultados: Tras haber estudiado a 48 pacientes diagnosticados de carcinoma oral estadio I-II (34 hombres y 14 mujeres, con edad media de 50 años), a los que se ha realizado la disección cervical, se han detectado adenopatías metastásicas en el 30\%. Con nuestros criterios utilizados para la detección de metástasis por ecografía, hemos obtenido una sensibilidad de hasta el 0,93 y un especificidad de hasta el 0,91, en función de los criterios utilizados.

๑ 2010 SECOM. Publicado por Elsevier España, S.L. Todos los derechos reservados.

\footnotetext{
*Autor para correspondencia.

Correo electrónico: Tesicreo@gmail.com (Teresa Creo Martínez).
} 


\title{
Ultrasound characteristics of malignant sub-clinical cervical lymph nodes in patients with oral epidermoid carcinoma
}

\begin{abstract}
A B S T R A C T
Oral carcinoma of oral cavity spread subclinical neck metastasis in $30-40 \%$, this situation justify elective neck dissection in treatment. The availability of diagnostic test that allow detect neck metastasis would avoid overtreatment in $60-70 \%$ of patients with cost and morbidity associated. Neck ultrasound detect subclinical metastasis with different sensitivity and specificity.

Objectives: Use ultrasound for detect metastatic lymph nodes before surgical treatment.

Materials and method: We make a prospective follow up of patients with diagnosis squamous carcinoma of oral cavity in stage I-II in our services. We make an ultrasound study (with long wave) before surgery treatment. We check and apply different malignant criteria that we get from literature. We compare ultrasound information with pathological results of cervical dissection, this is our gold standard for metastasis.

Results: We studied 48 patients with diagnostic of oral carcinoma in stage I-II, 34 men and 14 women with medium age of 50 years old. We make neck dissection, and we find metastatic lymph nodes in $30 \%$, with our criteria for detection of metastatic lymph node with ultrasound we obtain a sensitivity until 0.93 and a specificity until 0.91 depend on used criteria.
\end{abstract}

○ 2010 SECOM. Published by Elsevier España, S.L. All rights reserved.

\section{Introducción}

Las metástasis cervicales son el factor pronóstico fundamental en los pacientes con carcinoma epidermoide de cavidad oral $^{1,2}$. La supervivencia a 5 años en pacientes sin afectación ganglionar es del 75\%, mientras que si tienen un ganglio afectado es del $49 \%$, y del $30 \%$ si son dos las metástasis ${ }^{3}$.

La exploración clínica del cuello tiene escaso valor para determinar el estadio ganglionar real del carcinoma escamoso, ya que en este tipo de carcinoma se producen metástasis cervicales subclínicas en el $20-30 \%$ de los pacientes ${ }^{4}$, lo que justifica la disección cervical electiva o profiláctica dentro del tratamiento estándar. Esta disección permite una estadificación correcta del cuello, un tratamiento precoz de las metástasis y una correcta terapéutica adyuvante postoperatoria, según el resultado anatomoclínico resultante de la disección cervical. Sin embargo, implica un sobretratamiento del $60-70 \%$ de pacientes que no la necesitan, con el consecuente incremento de costes y de morbilidad.

Existen autores (Leemans et al, 2002) que en tumores en estadio I-II no agresivos ${ }^{5}$ defienden extirpar el tumor y esperar a ver la evolución de la enfermedad mediante seguimiento estricto $^{6}$, mientras que otros (Byers et al, 1998) defienden la realización de la disección cervical profiláctica ${ }^{7}$, sin que exista consenso respecto a los efectos de ambas decisiones en la supervivencia del paciente $\mathrm{e}^{8-10}$.

La disponibilidad de pruebas diagnósticas que detecten las metástasis subclínicas previamente al tratamiento sin necesidad de disección cervical profiláctica nos permitirían tomar decisiones respecto al tratamiento de los pacientes con cierta seguridad, asumiendo los menores errores y riesgos.

La evaluación del cuello mediante ultrasonidos nos permite detectar, visualizar y localizar las adenopatías cervicales, valorar si son metastásicas, y todo ello con la ventaja de su bajo coste e inocuidad.
La ecografía cervical detecta metástasis cervicales subclínicas en pacientes con cáncer oral en estadio I-II con una sensibilidad que varía entre el 60 y el $90 \%$ y una especificidad entre el 70 y el 90\%, dependiendo de los criterios diagnósticos ecográficos de malignidad utilizados ${ }^{11-14}$.

El objetivo de nuestro estudio es valorar la eficacia de la ecografía para el diagnóstico de metástasis cervicales subclínicas en el cáncer oral, utilizando diferentes criterios de malignidad, y si dicha eficacia puede mejorar combinando o asociando dichos criterios según el nivel de la adenopatía.

\section{Material y método}

Estudio de seguimiento prospectivo iniciado en el año 2002, de pacientes pertenecientes a nuestras áreas sanitarias, diagnosticados y tratados por carcinoma epidermoide de cavidad oral en estadio precoz (I-II) en nuestros servicios, a los que realizamos ecografía cervical previa al tratamiento.

El tratamiento quirúrgico consiste en todos los casos en extirpación de la lesión, seguida de disección cervical electiva unilateral si la lesión afecta a un solo lado de la cavidad oral, disección cervical electiva bilateral si afecta a la línea media, o de seguimiento y vigilancia clínica cervical en los pacientes en quienes la cirugía cervical profiláctica se considera que incrementa el riesgo para el paciente por su deterioro del estado físico general.

En los casos en que el estudio anatomopatológico confirma la afectación cervical metastásica y/o la afectación de bordes, se administra radioterapia postoperatoria.

Previo al tratamiento quirúrgico primario, a los pacientes se les realiza una ecografía cervical, estudiando ambos lados del cuello. Para ello utilizamos el ecógrafo Philips ATL versión HDI 4000, con sonda lineal entre 6-12 $\mathrm{mHz}$. 


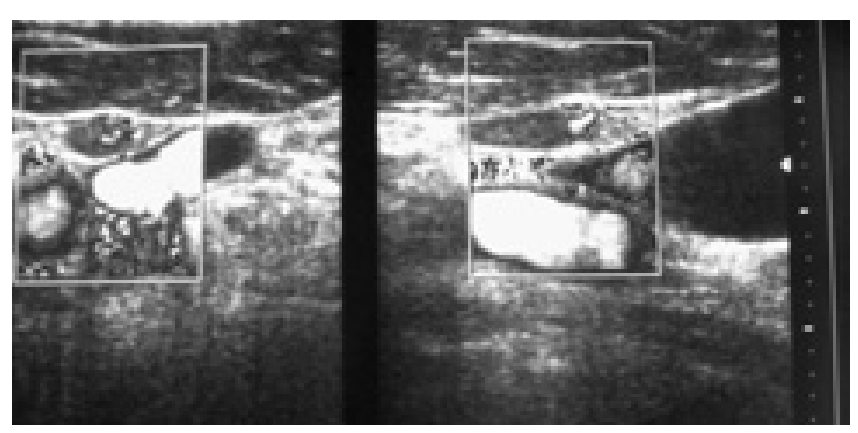

Figura 1 - Vascularización cortical de la adenopatía.

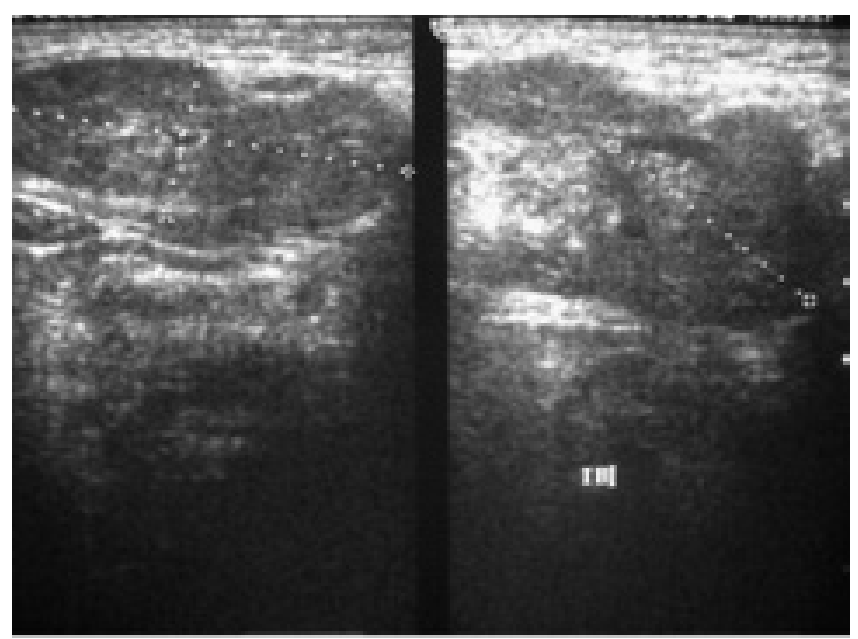

Figura 2 - Heterogenicidad de la adenopatía.

El estudio ecográfico es realizado por el mismo radiólogo con el mismo aparato, y consiste en detectar, localizar según el nivel ganglionar, medir en las tres dimensiones del espacio (transversal, longitudinal y anteroposterior) y valorar diferentes criterios ecográficos de malignidad de todas las adenopatías.

Los criterios ecográficos de malignidad se seleccionan de la revisión bibliográfica a fin de que sean los más fiables y reproducibles y los de mayor sensibilidad (porcentaje para detectar metástasis en el enfermo metastásico).

Los criterios seleccionados son:

- Vascularización cortical de la adenopatía y borramiento del híleo ${ }^{11,12}$ (fig. 1).

- Heterogenicidad de la adenopatía (fig. 2). Implica no homogenicidad de eco en su interior, y se visualiza con zonas hipecogénicas e hiperecogénicas ${ }^{14}$.

- Diámetro transverso o menor, sospechoso si supera los 7 $\mathrm{mm}^{13,15}$

- Cociente entre diámetro mayor (longitud) y menor (anchura). Se considera sospechoso de malignidad si es $<1,3 \mathrm{~mm}^{16}$. Este criterio se relaciona con la forma de la adenopatía, ya que al ser menor la relación, indica que es más redondeada.

Escogemos estos valores porque la mayoría de las adenopatías normales en cabeza y cuello son alargadas y con un diámetro de 2-5 $\mathrm{mm}^{15}$.
Todas las ecografías son valoradas según estos criterios diagnósticos y archivadas según protocolo de trabajo en una base de datos. Los datos recogidos son: paciente, filiación, datos clínicos del tumor, ecografía (número de ganglios, localización y si cumple alguno de los criterios malignos) y datos anatomoclínicos.

El patrón oro que utilizamos para identificar de manera correcta las metástasis cervicales es la confirmación anatomopatológica de las metástasis cervicales en las piezas de disección cervical y/o la aparición de metástasis confirmadas por biopsia o por punción-aspiración con aguja fina (PAAF) durante el seguimiento de los pacientes durante un período de 24 meses $^{3}$, tiempo en el que se detectan el 95\% de las metástasis cervicales. Cada paciente es considerado positivo o negativo en función de que presente o no alguna metástasis, independientemente del número y de la localización.

Debido a la dificultad de identificar y asignar en la pieza de disección cada adenopatía, con su valoración ecográfica, y dado que la decisión terapéutica de la disección se basaría en encontrar una sola adenopatía sospechosa, la detección del criterio de malignidad estudiado hace que sea el paciente, y no la adenopatía individual, lo que se clasifique como metástasis positivo o negativo.

Los resultados obtenidos con los diferentes criterios ecográficos de malignidad son comparados con el patrón oro para determinar la cifra de aciertos y errores, y valoramos la eficacia diagnóstica de los criterios mediante la sensibilidad, la especificidad, el valor predictivo positivo, el valor predictivo negativo y la razón de verosimilitud.

Con el objetivo de mejorar la eficacia diagnóstica, los criterios se combinarán en paralelo (criterio 1 o criterio 2) y en serie (criterio 1 y criterio 2), y variaremos los criterios y su combinación según el nivel de la adenopatías.

En el nivel II se considera positiva si el diámetro transversal es mayor de 7 y/o la relación longitud/anchura es menor de 1,3 ( $\mathrm{T}>7 \mathrm{y} / \mathrm{o} \mathrm{L} / \mathrm{T}<1,3$ ). Se asocian estos dos criterios en paralelo para aumentar la sensibilidad. Se establecen los $7 \mathrm{~mm}$ como límite de diámetro transverso porque es el consenso adoptado en la literatura como límite superior de la zona transversal de la adenopatía dentro de la normalidad.

En cualquiera de los otros niveles, para ser consideradas positivas tienen que cumplir que el diámetro transversal sea mayor de 7 y la relación de longitudes longitudinal/transversal sean menores de 1,3 $(\mathrm{T}>7 \mathrm{y} \mathrm{L} / \mathrm{T}<1,3)$. Combinamos estos dos criterios en serie para aumentar la especificidad, porque en este caso, si no se detecta alguna adenopatía que finalmente sea positiva, cuando aparezca, en estas regiones se puede abordar quirúrgicamente.

También valoramos la combinación de flujo vascular y el aspecto en una misma adenopatía, aumentando así la especificidad del estudio.

\section{Resultados}

En nuestro ensayo hemos estudiado a 48 pacientes desde el año 2002, diagnosticados de carcinoma epidermoide en cavidad oral estadio I-II. De ellos, son 34 hombres y 14 mujeres, con 
edades comprendidas entre los 30 y los 80 años (media, 55 años) y un seguimiento máximo de 4 años y mínimo de 1 año.

Realizamos 39 disecciones cervicales unilaterales y 5 bilaterales, y extirpación y seguimiento a 4 pacientes. De todos ellos, encontramos adenopatías metastásicas en el 34\% de los pacientes estudiados; es decir, que 14 pacientes de los estudiados que mostraban un NO clínico, finalmente presentaron metástasis en el estudio anatomopatológico.

Los resultados obtenidos, considerando los criterios cada uno por separado, se expresan en las tablas.

En la tabla 1 se exponen los pacientes con metástasis, donde se evalúan los casos que se han confirmado en el estudio anatomopatológico que son positivos. En la tabla 2 figuran los pacientes que no hacen metástasis, y se especifican los pacientes que han sido auténticos NO tras efectuar el estudio anatomopatológico.

Los resultados obtenidos se muestran en la tabla 3, y la sensibilidad y la especificidad se exponen en la tabla 4, mediante la aplicación de los distintos criterios expuestos.

En la tabla 3 se aprecia que siguiendo únicamente el criterio 1 , tenemos una sensibilidad muy alta, pero la especificidad es baja, mientras que con el criterio 2, más restrictivo, disminuimos la sensibilidad pero aumentamos la especificidad.

El tercer y cuarto criterios se refieren a la localización de las adenopatías, por lo que se restringe la valoración del cuello y justifica que, al no valorar otros niveles, pasen inadvertidas adenopatías que pueden ser metastásicas, disminuyendo la sensibilidad y aumentando la especificidad, sobre todo el criterio 4, que hace la combinación en serie.

El criterio 3 se refiere a adenopatías en el nivel II. Para considerarla positiva se precisa que el diámetro transversal sea
$>7 \mathrm{~mm}$, y/o la relación $\mathrm{L} / \mathrm{T}<1,3$. Esta diferencia que hacemos en el nivel II se debe a la dificultad del abordaje quirúrgico de este nivel, de manera que si erramos, la posibilidad de extirpar la metástasis es poca, por lo que aquí optamos por correr los menos riesgos posibles.

El criterio 4 se refiere a los otros niveles estudiados, donde la consideramos positiva si cumple ambos criterios $(\mathrm{T}>7$ y $\mathrm{L} / \mathrm{T}<1,3)$, aumentando así la especificidad, ya que estos niveles son fácilmente abordables quirúrgicamente si se detectan los cambios a tiempo.

Con la combinación de los criterios 3 y 4, donde los criterios se aplican según el nivel de la localización, obtenemos una sensibilidad de 0,93 y sólo un falso negativo, es decir, detectamos casi todas las metástasis y con una especificidad de 0,76 , lo cual es muy asumible.

El criterio 5 se refiere a la combinación de aspecto y vascularización. Por sí solo no arroja muy buenos resultados, pero combinado con los otros criterios nos da una idea de la malignidad de la adenopatía en caso de duda.

\section{Discusión}

La presencia de adenopatías cervicales metastásicas es un factor fundamental para el pronóstico del paciente y para la planificación del tratamiento ${ }^{14}$. Sin embargo, su detección puede ser dificultosa. Podemos encontrar adenopatías palpables, que finalmente son nódulos reactivos, sin afectación metastásica, y por otro lado, en cuellos clínicamente negativos podemos encontrar adenopatías subclínicas positivas. Adicionalmente, la realización de la disección cervical en los

Tabla 1 - Pacientes tras el estudio anatomopatológico

\begin{tabular}{|c|c|c|c|c|c|}
\hline & Criterio 1 & Criterio 2 & Criterio 3 & Criterio 4 & Criterio 5 \\
\hline & $\mathrm{T}>7 \mathrm{~mm}$ & $\mathrm{~L} / \mathrm{T}<1,3 \mathrm{~mm}$ & $\begin{array}{l}\text { Nivel II T }>7 \mathrm{~mm} \\
\text { o LT }<1,3 \mathrm{~mm}\end{array}$ & $\begin{array}{c}\text { Otros niveles } \mathrm{T}>7 \mathrm{~mm} \\
\mathrm{y} \mathrm{L} / \mathrm{T}<1,3 \mathrm{~mm}\end{array}$ & $\begin{array}{c}\text { Aspecto y } \\
\text { vascularización }\end{array}$ \\
\hline Paciente 1 & + & - & + & - & + \\
\hline Paciente 2 & + & + & - & + & + \\
\hline Paciente 3 & + & + & + & + & - \\
\hline Paciente 4 & + & + & + & + & + \\
\hline Paciente 5 & + & + & + & + & - \\
\hline Paciente 6 & + & + & + & + & + \\
\hline Paciente 7 & + & - & - & - & + \\
\hline Paciente 8 & + & + & + & + & + \\
\hline Paciente 9 & + & + & + & + & + \\
\hline Paciente 10 & + & + & + & - & + \\
\hline Paciente 11 & + & + & + & + & + \\
\hline Paciente 12 & - & + & + & - & + \\
\hline Paciente 13 & + & + & + & + & - \\
\hline Paciente 14 & + & + & - & + & + \\
\hline Falsos negativos & $1 / 14$ & $2 / 14$ & $3 / 14$ & $4 / 14$ & $3 / 14$ \\
\hline \multicolumn{6}{|c|}{$\begin{array}{l}\text { La tabla muestra a los pacientes en los que se ha confirmado tras el estudio anatomopatológico la existencia de metástasis, si se han detectad } \\
\text { estas metástasis según los criterios que hemos aplicado tras realizar la ecografía. Criterio } 1 \text { es el diámetro transversal de la adenopatía. Criter } \\
2 \text { es el cociente entre la longitud y el diámetro transversal. Criterio } 3 \text { se considera positivo para el nivel II si el diámetro transversal es superic } \\
\text { a } 7 \mathrm{~mm} \text { o si el cociente entre la longitud y el diámetro transversal es inferior a } 1,3 \mathrm{~mm} \text {. Criterio } 4 \text { se considera positivo para otros niveles si } \\
\text { diámetro transversal es superior a } 7 \mathrm{~mm} \text { y si el cociente entre la longitud y el diámetro transversal es inferior a } 1,3 \text { mm. Criterio } 5 \text { valora d } \\
\text { forma positiva la heterogenicidad, y el íleo vascular. }\end{array}$} \\
\hline
\end{tabular}


casos sin metástasis aumenta la morbilidad de estos pacientes, que en muchos casos son de elevada edad, con patología asociada, y por tanto con elevado riesgo para someterse a este tipo de intervenciones.

Por todo ello es evidente la importancia de encontrar un medio eficaz y fiable que nos permita valorar de manera preoperatoria el cuello en pacientes con carcinoma epidermoide, para así no tener que someterlos a una disección cervical innecesaria con todos los riesgos y costes que implica, sin dejar de operar los casos que presenten verdaderas metástasis subclínicas.

Los criterios ecográficos que hemos empleado y su combinación han demostrado una elevada sensibilidad en la detección de adenopatías metastásicas, con un bajo número de falsos negativos, que son los que debemos evitar, con el fin de seguir realizando un tratamiento precoz de dichas metástasis sin afectar a la supervivencia.

Aunque tenemos una buena especificidad, de hasta el 91\% en función de los criterios combinados, puede ser menor que la obtenida en otros trabajos, puesto que para nosotros en este momento lo más importante es la sensibilidad, por las consecuencias que tienen los falsos negativos.

Hay autores, como Yusa et $\mathrm{al}^{14}$, que en un estudio de 41 pacientes presentan una sensibilidad de $82 \%$ y una especificidad del 91\%, y que defienden la realización de una ecografía preoperatoria para la selección de pacientes, aplicando una ecuación de regresión lógica. Asimismo, Hayashi et al ${ }^{17}$ defienden el seguimiento de pacientes con un TI o TII con ecografía; tras estudiar a 18 pacientes se obtuvieron muy buenos resultados, e incluso en los pacientes que presentaron

\section{Tabla 2 - Pacientes sin adenopatías metastásicas tras el estudio por el patólogo}

\begin{tabular}{|c|c|c|c|c|c|}
\hline & Criterio 1 & Criterio 2 & Criterio 3 & Criterio 4 & Criterio 5 \\
\hline & $\mathrm{T}>7 \mathrm{~mm}$ & $\mathrm{~L} / \mathrm{T}<1,3 \mathrm{~mm}$ & $\begin{array}{l}\text { Nivel II T > } 7 \mathrm{~mm} \\
\text { o LT }<1,3 \mathrm{~mm}\end{array}$ & $\begin{array}{c}\text { Otros niveles } \mathrm{T}>7 \mathrm{~mm} \\
\mathrm{y} \mathrm{L} / \mathrm{T}<1,3 \mathrm{~mm}\end{array}$ & $\begin{array}{c}\text { Aspecto y } \\
\text { vascularización }\end{array}$ \\
\hline Paciente 15 & - & - & - & - & - \\
\hline Paciente 16 & - & - & - & - & - \\
\hline Paciente 17 & + & - & - & - & - \\
\hline Paciente 18 & - & + & - & - & + \\
\hline Paciente 19 & + & - & - & - & - \\
\hline Paciente 20 & + & - & - & - & + \\
\hline Paciente 30 & - & - & - & - & + \\
\hline Paciente 31 & + & - & + & - & + \\
\hline Paciente 32 & - & - & - & - & + \\
\hline Paciente 33 & - & - & - & - & - \\
\hline Paciente 34 & - & - & - & - & + \\
\hline Paciente 35 & - & - & - & - & - \\
\hline Paciente 36 & - & - & - & - & - \\
\hline Paciente 37 & + & - & - & - & - \\
\hline Paciente 38 & - & - & - & - & + \\
\hline \multicolumn{6}{|l|}{ Paciente 39} \\
\hline Paciente 40 & - & - & - & - & - \\
\hline Paciente 41 & + & - & - & - & - \\
\hline Paciente 42 & - & - & - & - & - \\
\hline Paciente 43 & + & + & + & + & - \\
\hline Paciente 44 & - & - & - & - & - \\
\hline Paciente 45 & - & - & - & - & - \\
\hline Paciente 46 & - & - & - & - & - \\
\hline Paciente 47 & + & - & - & - & + \\
\hline Paciente 48 & - & - & - & - & - \\
\hline Paciente 49 & - & - & - & - & + \\
\hline Paciente 50 & + & + & + & + & - \\
\hline Paciente 51 & - & + & - & - & - \\
\hline Paciente 52 & + & - & + & - & + \\
\hline Paciente 53 & + & - & + & - & - \\
\hline Paciente 54 & + & + & + & + & + \\
\hline Paciente 55 & + & - & + & - & - \\
\hline Paciente 56 & - & + & + & - & + \\
\hline Paciente 57 & - & - & - & - & - \\
\hline Verdaderos negativos & $21 / 34$ & $28 / 34$ & $26 / 34$ & $31 / 34$ & $21 / 34$ \\
\hline
\end{tabular}

Se exponen los pacientes que tras el estudio por el patólogo no presentaron adenopatías metastásicas, y los resultados obtenidos en el estudio ecográfico previo tras la aplicación de los criterios expuestos. Criterio 1 es el diámetro transversal de la adenopatía. Criterio 2 es el cociente entre la longitud y el diámetro transversal. Criterio 3 se considera positivo para el nivel II si el diámetro transversal es superior a 7 mm o si el cociente entre la longitud y el diámetro transversal es inferior a 1,3 mm. Criterio 4 se considera positivo para otros niveles si el diámetro transversal es superior a $7 \mathrm{~mm}$ y si el cociente entre la longitud y el diámetro transversal es inferior a 1,3 mm. Criterio 5 valora de forma positiva la heterogenicidad y el íleo vascular. 
Tabla 3 - Sensibilidad y especificidad calculada para cada criterio por separado, en función de los datos presentados en las tablas 1 y 2

\begin{tabular}{lccccc}
\hline & Criterio 1 & Criterio 2 & Criterio 3 & Criterio 4 & Criterio 5 \\
\cline { 2 - 6 } & T $>$ 7 mm & L/T $<1,3 \mathrm{~mm}$ & $\begin{array}{c}\text { Nivel II T }>7 \mathrm{~mm} \\
\text { o LT }<1,3 \mathrm{~mm}\end{array}$ & $\begin{array}{c}\text { Otros niveles T }>7 \mathrm{~mm} \\
\text { y L/T }<1,3 \mathrm{~mm}\end{array}$ & $\begin{array}{c}\text { Aspecto y } \\
\text { vascularización }\end{array}$ \\
\hline Sensibilidad & 0,93 & 0,86 & 0,78 & 0,71 & 0,78 \\
Especificidad & 0,62 & 0,82 & 0,76 & 0,91 & 0,62 \\
VPP & 0,5 & 0,67 & 0,57 & 0,76 & 0,46 \\
VPN & 0,95 & 0,93 & 0,89 & 0,88 & 0,87 \\
RV+ & 2,4 & 4,78 & 3,25 & 7,8 & 2,63 \\
RV- & 0,11 & 0,17 & 0,29 & 0,32 & 0,35 \\
\hline
\end{tabular}

RV: razón de verosimilitud; VPN: valor predictivo negativo; VPP: valor predictivo positivo.

En esta tabla se expone la sensibilidad y especificidad calculada para cada criterio por separado, en función de los datos presentados en las tablas 1 y 2.

\section{Tabla 4 - Sensibilidad y especificidad calculadas para los criterios ya expuestos de forma combinada}

\begin{tabular}{lcccc}
\hline & Criterios 3 y 4 & Criterios 3 y 4 y & Criterios 1 y 2 & Criterios 1 o 2 \\
\hline Falsos & $1 / 14$ & 0 & $3 / 14$ & 0 \\
Verdaderos & $26 / 34$ & $17 / 34$ & $31 / 34$ & $18 / 34$ \\
Sensibilidad & 0,93 & 1 & 0,78 & 1 \\
Especificidad & 0,76 & 0,5 & 0,91 & 0,53 \\
\hline
\end{tabular}

recidiva locorregional, la disección cervical fue curativa. Otros autores que defienden la ecografía cervical preoperatoria son Nieuwenhuis y Hodder ${ }^{3,6}$; en adenopatías dudosas asocian la PAAF a la ecografía, y sólo realizan disección cervical si los criterios ecográficos lo indican; Nieuwenhuis et al presentan el $21 \%$ de pacientes con recurrencias locorregionales, a los que se realiza disección cervical, y el $88 \%$ de pacientes libres de enfermedad tras disección cervical; Hodder et al sólo realizan disección cervical cuando las adenopatías son claramente malignas en la ecografía y PAAF en caso de duda, teniendo el $6 \%$ de falsos negativos. Bondt et $\mathrm{al}^{19}$ presentan un metaanálisis donde revisan la bibliografía sobre la detección precoz de adenopatías cervicales ocultas en el carcinoma epidermoide de cavidad oral mediante distintas técnicas de imagen. Su grupo analiza la sensibilidad, la especificidad, los falsos positivos y los falsos negativos según las distintas técnicas de imagen, llegando a la conclusión de que la ecografía guiada por PAAF es la técnica más eficaz para la detección de metástasis ocultas, llegando a una sensibilidad del 90\% y una especificidad del 100\%, mientras que otras técnicas de imagen, como la tomografía computarizada (TC), presentan una sensibilidad en torno al $60 \%$ y una especificidad en torno al $65 \%$, y la resonancia magnética (RM), una sensibilidad y especificidad muy similares a las de la TC, presentando como única ventaja la baja variación interobservador, que es mayor en la ecografía ${ }^{19}$.

Por otro lado, hay autores que defienden la disección cervical a pesar de las posibles complicaciones. Tal es el caso de Haberal et $\mathrm{al}^{15}$, que presenta un estudio de 48 pacientes a los que se realiza una ecografía preoperatoria que ofrece resulta- dos con una sensibilidad del $72 \%$, una especificidad del $96 \%$, valor predictivo positivo del $94 \%$ y valor predictivo negativo del $80 \%$. En la misma línea está T. Stuckensen ${ }^{18}$, que presenta un estudio de 106 pacientes con una sensibilidad del $84 \%$ y una especificidad del $68 \%$. Aunque las muestras son grandes, estos autores no valoran los pacientes en sí, sino las adenopatías, sin tener en cuenta ni el nivel, ni otros datos. Nosotros, sin embargo, planteamos valorar a los pacientes en conjunto, es decir, valorar el riesgo de la adenopatía según la localización, siendo más estrictos en niveles más difíciles de abordar quirúrgicamente. Además no consideran la iatrogenia originada por la disección cervical, como en el caso de Po Wing Yuen et $\mathrm{al}^{20,21}$, que presentan un estudio de 50 pacientes en los cuales incluye No clínico, pero no siempre radiológico. Tras realizar desde disecciones suprahomohioideas ${ }^{22}$ hasta disecciones radicales, se detectan 11 recurrencias y un 3\% de recurrencias contralaterales, además de una muerte por complicaciones postoperatorias, por lo que hay que tener en cuenta todos estos inconvenientes en la realización de la disección cervical.

En la misma línea está Byers et $\mathrm{al}^{7}$, que presentan un estudio de 91 pacientes a los cuales sólo realizan ecografías a 39 de ellos. Además en su estudio incluyen a pacientes desde T2 a T4 y N0, N1 y N2, realizan un modelo de regresión multivariable y, según sus datos, los pacientes deben tener una disección cervical electiva, aunque admiten que se necesitan otros estudios que lo corroboren. Lo mismo puede decirse de la disección cervical electiva que presentan Hodder et $\mathrm{al}^{3}$, que critican que en la disección electiva se realizan los niveles I y II, mientras que un número importante de pacientes presen- 
tan la metástasis en nivel III y IV sin haberla presentado en los niveles I y II.

Después de los resultados expuestos, consideramos que es alentador, y que puede considerarse una prueba de futuro, el estudio cervical preoperatorio de estos pacientes, y aunque actualmente no hemos terminado nuestro estudio - ya que seguimos añadiendo pacientes, para poder así aumentar la fiabilidad estadística-, los resultados obtenidos hasta el momento son muy esperanzadores en vistas a evitar la disección cervical a un importante número de pacientes, lo que nos anima a seguir el estudio en esta línea de investigación.

Debido a su bajo coste e inocuidad, se puede seguir a los pacientes sin disección cervical y se puede realizar cirugía precoz de rescate. Nuestro protocolo es más restrictivo con las adenopatías situadas en el nivel II, ya que son las de mayor dificultad de abordaje, y además entrañan un mayor riesgo de extensión a la base del cráneo, lo que dificulta entonces el control de la enfermedad, en caso de que durante el seguimiento se detecte precozmente alguna metástasis. Además, la ecografía nos permite realizar un seguimiento exhaustivo y abordar el problema de manera rápida en caso de que ocurra ${ }^{16}$. La ecografía es, pues, un método factible y eficaz para el seguimiento de estos pacientes y para la detección precoz de adenopatías metastásicas en caso de que aparezcan, por lo que pueden ser abordadas rápidamente, como ya lo hacen tanto Hodder et al como Nieuwenhuis et al en sus áreas hospitalarias.

\section{Conclusión}

Hemos visto que la ecografía puede ser una prueba válida debido a su elevada sensibilidad y especificidad en el estadificación del carcinoma epidermoide de cavidad oral en estadio I-II y cuellos negativos, porque es útil para detectar metástasis ocultas, permitiéndonos reducir las disecciones cervicales electivas innecesarias.

\section{B I B L I O G R A F Í A}

1. Davis RK, Stoker K. Prognostic indicators in head and neck cancer patients receiving combined therapy. Arch Otolaryngol Head Neck Surg. 1989;115:1443-6.

2. Munck J, Cwitkovic EJ, Piekarski D. Computed tomographic density of metastatic lymph nodes as a treatment-related prognostic factor in advanced head and neck cancer. 1991;83:569-75.

3. Hodder SC, Evans RM, Patton DW, Silvester KC. Ultrasound and fine needle aspiration cytology in the staging of neck lymph nodes in oral squamous cell carcinoma. Br J Oral Maxillofac Surg. 2000;38:430-6.

4. Pitman K, Johnson J, Myers E. Effectiveness of selective neck dissection for management of the clinically negative neck. Arch Otolaryngol Head Neck Surg. 1997;123:917-22.

5. Nieuwenhhuis EJC, Colnot DR, Pijpers JA, Castelijns HJ, van Diest PJ, Brakenhoff $\mathrm{RH}$, et al. Lymphoscintigraphy and ultrasound-guided fine needle aspiration cytology of sentinel lymph nodes in head and neck cancer patients. Recent Results Cancer Res. 2000;157:206-17.

6. Nieuwenhuis E, Castelijns J, Pijpers R, van den Bekel M, Brakenhoff R, van der Waal I, et al. Wait-and-see policy for the
NO neck in early-stage oral and oropharyngeal squamous cell carcinoma using ultrasonography-guided cytology: is there a role for identification of the sentinel node? Head \& Neck. 2002;24:282-9.

7. Byers R, El-Naggar A, Yen Lee Y, Rao B, Fornage B, Ferry N, et al. Can we detect or predict the presence of occult nodal metastases in patients with squamous carcinoma of the oral tongue? Head \& Neck. 1998;20:138-44.

8. Vandenbrouck C, Sancho-Garnier H, Chassagne D, Saravane D, Cachin Y, Micheau C. Elective versus therapeutic radical neck dissection in epidermoid carcinoma of the oral cavity. Cancer. 1980;46:386-90.

9. Fakih A, Rao RS, Borges A, Patel AR. Elective versus therapeutic neck dissection in early carcinoma of the oral tongue. Am J Surg. 1989;158:309-13.

10. Khafif RA, Gelbfish GA, Attie JN, Tepper P, Zingale R. Thirty year experience with 457 radical neck dissections in cancer of the mouth, pharynx, and larynx. Am J Surg. 1989;158:303-8.

11. Yusa $H$, Yoshida $H$, Iwasa $S$, Ueno E. Ultrasonographic assessment for response to radiochemotherapy of metastatic cervical lymph nodes in head and neck cancer: usefulness of grey-scale and color Doppler sonography. Ultrasound in Medicine and Biology. 2000,26:1081-7.

12. Ahuja A, Ying M. Grey-scale sonography in assessment of cervical lymphadenopathy: review of sonographic appearances and features that may help a beginner. British Journal of Maxillofacial Surgery. 2000;38:451-9.

13. Gritzmann N, Hollerweger A, Macheiner P, Rettenbacher $T$. Sonography of soft tissue masses of the neck. Clin Ultrasound. 2002;30:356-73.

14. Yusa $H$, Yoshida $H$, Ueno E. Ultrasonographic criteria for diagnosis of cervical lymph node metastasis of squamous cell carcinoma in the oral and maxillofacial region. J Oral Maxillofac Surg. 1999;57:41-8.

15. Haberal I, Çelik H, Göçmen H, Akmansu H, Yörük M, Özeri C. Which is important in the evaluation of metastatic lymph nodes in head and neck cancer: Palpation, ultrasonography, or computed tomography? Otolaryngol Head Neck Surg. 2004;130:197-201.

16. Koischwitz D, Gritzmann N. Ultrasound of the neck. Radiol Clin North Am. 2000;38:1029-45.

17. Hayashi T, Ito J, Haira S, Katsura K, Shingaki S, Hoshina H. The clinical significance of follow-up sonography in the detection of cervical lymph node metastases in patients with stage I or II squamous cell carcinoma of the tongue. Oral Surg Oral Med Oral Pathol Oral Radiol Endod. 2003;96:112-7.

18. Stuckensen T, Kovács A, Adams S, Baum R. Staging of the neck in patients with oral cavity squamous cell carcinomas: a prospective comparison of PET, ultrasound, CT and MRI. J Craniomaxillofac Surg. 2000;28:319-24.

19. Bondt RBJ, Nelemans PJ, Hofman PAM, Casselman JW, Kremer B, van Engelshoven JMA, et al. Detection of lymph node metastases in head and neck cancer: A meta-analysis comparing US, USgFNAC, CT and MR imaging. Eur J Radiol. 2007;64:266-72.

20. Po Wing Yuen A, Wei WI, Ming Wong Y, Chi Tang K. Elective neck dissection versus observation in the treatment of early oral tongue carcinoma. Head \& Neck.1997;19:583-8.

21. Po Wing Yuen A, Yin Lam K, Chak Lam Chan A, Ignace Wei W. Clinicopathological analysis of elective neck dissection for N0 neck of early oral tongue carcinoma. Am J Surg. 1999;177:90-2.

22. Brazilian Head and Neck Cancer Study Group. Results of a prospective trial on elective modified radical classical versus supraomohyoid neck dissection in the management of oral squamous carcinoma. Am J Surg. 1998;176:422-7. 